\title{
Supporting a Multidisciplinary Digital Media Research Community with GRAND Aspirations
}

\author{
Kellogg S. Booth ${ }^{1}$ and Eleni Stroulia ${ }^{2}$ \\ ${ }^{1}$ Computer Science, The University of British Columbia, Vancouver, BC V6T 1 Z4 Canada \\ ${ }^{2}$ Computing Science, University Alberta, Edmonton, AB T6G 2E8, Canada \\ ksbooth@cs.ubc.ca, stroulia@ualberta.ca
}

\begin{abstract}
The challenges of managing a nationwide "network of centres of excellence" (NCE) are being explored by GRAND, a Canadian NCE comprising over 350 researchers from technical disciplines, social sciences, humanities and the arts within 34 interwoven projects focusing on all aspects of digital media. A complex web of relationships with funding agencies, private and public partners, and researchers is being managed using a purpose-built web-based platform (the GRAND Forum) that supports communication and collaboration across communities. The Forum explicitly represents multiple roles of individuals within the organization through formal and informal usercentred workflows that reflect both symmetric (peer-to-peer) and asymmetric (hierarchical) organizational structures. We describe the principles of each.
\end{abstract}

Keywords: Interdisciplinary research collaboration.

Grand challenges such as climate change, energy shortage, the threat of pandemics, and dealing with the accelerating pace of cultural shifts in society are being tackled through large-scale, multidisciplinary research teams. Digital media in its many forms is a tool for these organizations, but is itself a challenge to be tackled. The Graphics, Animation, and New Media network of centres of excellence (GRAND) has multiyear funding from the Canadian federal government for five research themes: (1) new media challenges \& opportunities, (2) games \& interactive simulation, (3) animation, graphics \& imaging, (4) social, legal, economic \& cultural perspectives, and (5) enabling technologies \& methodologies. These span across disciplines, both public and private organizations, and provincial borders. Each theme has significant intersection with concerns familiar to the HCI community, and the management of the GRAND network faces problems well known in HCI for which are pursuing innovative solutions. We describe two aspects of our approach to these challenges.

\section{Management Structures to Promote Multidisciplinary Research}

Collaboration is "designed into" GRAND. Each of 34 projects has a project leader and co-leader from different organizations, often from different disciplines. Each of 64 principal network investigators (PNIs) is required to engage with multiple projects (three on average) and every project is expected to contribute to the goals of multiple themes. Projects are defined by a combination of top-down and bottom-up processes. 
Individual PNIs "invest" in projects by apportioning their funding amongst projects in which they engage. Theme leaders look for a balance across projects to advance the shared goals of the network. Funding to PNIs is based on contributions to those goals.

Information about the research is collected using a "report once, use often" strategy to minimize duplication of effort. Research activities, publications, prototypes and research artifacts are described by whoever first reports them and then re-used in subsequent reports. A wiki-like approach to maintaining information allows an initial reporter's information to be updated by others, and linked to all relevant projects, rather than requiring it be siloed into individual project reports. This raises mutual awareness and hopefully leads to serendipitous collaboration across projects.

Following Pascal's dictum "The stream is always purer at its source [Les choses valent toujours mieux dans leur source]," the reporting hierarchy is flattened. Those doing the actual research (most often students and postdocs) provide the initial reports, with supervisors, then project leaders, and finally theme leaders synthesizing summaries in which "raw data" can always be accessed by drilling down.

Two special projects, Media Enabled Organizational Workflow (MEOW) and Network Assessment and Validity for Effective Leadership (NAVEL), will support and reflect on the activities of the GRAND community in a yin-and-yang symbiosis as GRAND evolves through its lifecycle. A set of tools built on the Mediawiki platform is a key component of this organizational management strategy.

\section{The GRAND Forum: Scaffolding for Collaboration}

To streamline workflows and support communication and collaboration across the network, a web-based Forum is being developed that builds on the principles in the previous section. People in GRAND have roles that evolve over time. Explicit rolebased mechanisms manage individual's workflows and maintain a history of those roles (from graduate student to network investigator to industry sponsor). Access control policies associated with roles reflect a variety of concerns, including crossjurisdictional privacy legislation and IP ownership issues.

Activities such as entering information about a publication are simple single-step workflows. Others, such as reporting project milestones, involve the iterative collaborative editing of multiple pieces of information by multiple contributors, each with different reporting deadlines and degrees of editing authority. Those workflows are too complex to be specified simply at the user-interface level and are too human intensive to be "managed" by traditional workflow engines. We are developing a lightweight event-driven workflow specification and enactment web-service to address this requirement.

GRAND will be evaluated in part by whether its members increase interactions with each other over time. Tracking these relationships is at best tedious and at worst exhausting. We are developing a tagging approach - both interactive through the user interface and automatic through a text-analysis process - so that interactions with artifacts managed by the Forum will enable us to recognize communication channels between people and projects. Audit trails to substantiate information in the Forum and to enable effective use by all our members (subject to the access-control practices above) will be supported by full text and faceted search guided by the network's organizational structures, which are part of the scaffolding underlying the Forum. 